\title{
SARS-CoV-2 and regular patient treatment - from the use of rapid antigen testing up to treatment specific precaution measures
}

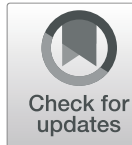

\author{
Jürgen Durner ${ }^{1,2^{*}}$, Thomas Beikler ${ }^{3}$, David C. Watts ${ }^{4}$, Marc Becker ${ }^{1,2}$ and Miriam E. Draenert ${ }^{1}$
}

\begin{abstract}
Introduction: The COVID-19 pandemic poses a continued challenge for all parties involved especially for the dentist as routine operation must be resumed. Rapid Antigen Tests (RATs) are actually recommended to identify and minimize infectious risks. However, there is still no guideline on the implementation of RATs in a dental or medical setting.
\end{abstract}

Methods: Based on data and an extensive literature research regarding rapid antigen testing and reflecting the recommendations given by the various professional societies a task force was formed to determine a specific testing and treatment strategy.

Results: A comprehensive test and treatment strategy and risk analysis was developed with practical suggestions for a wide range of typical activities in dental and medical offices.

The transmission of SARS-CoV-2 and its variants via aerosols and droplets as well as the difficulties to maintain the minimum distance form special challenges to the dental routine. RATs might in addition to optimal and necessary hygienic standards in combination with the use of adequate personal protection equipment be an important instrument in managing the challenges.

Conclusions: The present work gives recommendations for dental routine operation (dental practices, outpatient clinics) to provide the necessary dental care for the population while protecting the doctor, practice team and patient at the same time.

Keywords: COVID-19, SARS-CoV-2, Dental Treatment, Rapid Antigen Tests, Precaution, Dentistry, Dental Practice

\section{Introduction}

The spread of severe acute respiratory syndrome coronavirus 2 (SARS-CoV-2) is classified as a pandemic by the world health organization (WHO). Recently, there have been several variants whose contagiosity seems to be increased compared to the wild type, such as B.1.1.7 (20I/ 501Y.V1) known as the UK variant or B.1.351 $(20 \mathrm{H} /$

\footnotetext{
* Correspondence: Juergen.durner@med.uni-muenchen.de

'Department of Conservative Dentistry and Periodontology, University Hospital, LMU Munich, Ludwig-Maximilians-University Munich, Goethestr. 70, Goethestraße 70, 80336 Munich, Germany

${ }^{2}$ Laboratory Becker \& Colleagues, Führichstr. 70, 81671 Munich, Germany Full list of author information is available at the end of the article
}

501Y.V2) known as the South African variant. Close contact or short-range transmission of infectious saliva droplets is the primary mode for spreading SARS-CoV-2 [1]. Therefore, the dental team is at risk for infection since it is impossible to keep a minimum safe distance of $1.5 \mathrm{~m}$ during treatment or to use partition walls and the patients cannot wear face masks during treatment. However, this holds true for the patient as well, at least in part. Moreover, the use of water-cooled rotating, oscillating (sonic- or ultrasonic) and air-polishing devices can increase the risk for infection by generating pathogen-containing aerosols $[2,3]$. The highest 
concentrations of contaminated aerosols are found located at the nose, the head, the chest, the arms and the mouthguard of dentists and dental assistants [4]. Interestingly, those aerosols can be spread over a range of $30 \mathrm{~m}$ in an indoor setting, e.g. a dental office, indicating potential long-distance saliva aerosol transmission [5]. Air circulation by ventilation and insufficient air conditioning systems may worsen the effect.

While vaccination against SARS-CoV-2 began in late 2020 , the recent spread of more aggressive and potentially resistant variants may counteract vaccination effects and thus interfere with the reinstallation of a prepandemic dental routine [6].

Testing in this context is a practical tool for identifying potentially infected individuals. It allows not only to isolate and identify contact traces and thus reduce the spread, but also to select the appropriate measures concerning the handling of the respective individuals.

The analytical gold standard for the detection of the virus and some of the variants SARS-CoV-2 is the reverse transcription-quantitative polymerase chain reaction (RT-qPCR) from a nasopharyngeal swab or throat swab [7-9]. The RT-qPCR examination cannot, however, be carried out directly on the patient, since the sample must be transferred to the laboratory and the corresponding result of the analysis returned. The desire for a point of care (POC) testing system, independent of specialized staff, that allows on-site testing and a rapid result has led to the introduction of rapid antigen tests (RATs) [10]. However, the advantage of on-site testing via RATs is mitigated by lower sensitivity compared to RT-qPCR [7, 11]. Nevertheless, RATs can be administered by laypersons without specific training [12]. Thus, the use of RATs has been proposed in various contexts of daily life, such as in educational, working or recreational environments.

Surprisingly, no literature reference can be found on the implementation of RATs within the testing and treatment strategy of a dental practice. Therefore, the aim of this study was to develop a test and treatment strategy that enables a dental practice to function during periods of SARS-CoV-2 prevalence by safeguarding to a maximum safety level for both patients and the dental care team.

\section{Methods}

Following an extensive literature research, regarding rapid antigen testing and reflecting the recommendations given by the various professional societies, a joint group was formed to establish a specific testing and treatment strategy. The key word searches in PubMed and the Cochrane Library, for articles published up to March 2021, were combinations of: dentistry, dental surgery, dentist, dental, patient, corona, SARS-CcV-2, rapid antigen test, dental personal, influence test strategy.

\section{Results}

A comprehensive test strategy must be applicable to both patients and all dental staff.

\section{Dental Personal}

The dentist and his/her staff should undergo a basic triage.

1) For symptom-free dentists and dental staff a RAT is recommended, with a regularity dependent on the prevalence.

2) In suspicious cases RATs can be applied to identify a potential infection.

3) Should the dentist or his/her staff show any signs of typical symptoms such as fewer, cough, sore throat, hoarseness, diarrhea or have been in contact with a SARS-CoV-2 positive persons within the last two weeks they should stay at home and should be tested by RT-qPCR.

4) Should, after treatment, it be determined that a patient has a positive test result regarding SARS$\mathrm{CoV}-2$, all contact persons should be tested, dependent on the duration, type of treatment and protective measures in place. The steps to be taken should be in line with the requirements of the local authorities.

Asymptomatic carriers are of particular importance, as they can pass on the virus without becoming ill themselves and can contribute to the transmission of the virus [13]. This is particularly important because there are immunosuppressed patients, e.g. after organ transplantation, who cannot build up antibody protection despite vaccination [14]. To minimize the respective risk, it is recommended to test the dentist and his staff on a regular and frequent base. The frequency with which RAT are performed should be adjusted (twice or more a week) depending on the actual infection level, prevalence and be consistent with guidelines of the respective authorities.

\section{Patients}

It is recommended that all patients are triaged before any appointment (preferably by phone and prior to entering the dental office) to determine their SARS-CoV-2 status, i.e. whether they show any typical symptoms such as fever, cough, sore throat, hoarseness, diarrhea or have been in contact with SARS-CoV-2 positive persons within the last two weeks (suspected of SARS-CoV-2) or have been tested positive for SARS-CoV-2 [2]. 
Based on the information received the following patient groups can be identified:

A) patients without any signs of SARS-CoV-2 infection.

B) patients suspected of SARS-CoV-2 infection regardless of whether they have been already vaccinated or had COVID-19 previously.

C) patients tested positive for SARS-CoV-2.

When entering the dental office all patients of group A) should be invited to undergo a RAT. If such a patient refuses to take the test, a healthy carrier status cannot be excluded.

A RAT is strongly advised for patients of group B). If such a patient refuses to take a RAT, the patient should be regarded as if they tested positive.

For patients in groups A and B who refuse a RAT, the treatment-option benefits of a test should be explained.

Patients of group C) should not be re-tested.

Whenever the RAT is positive the patient should be isolated immediately, the necessary authorities contacted and an RT-qPCR-test completed. Only emergency treatments should be performed.

This general procedure is straightforward and flexible to apply and should maximize safety.

\section{Treatment strategy}

The treatment strategy should allow for the maximum of dental treatment possibilities given the respective risk assessment status of a SARS-CoV-2 infection.

The following considerations should be taken into account and adapted if necessary.

For patients of group A) who have a negative RAT and are without any signs of SARS-CoV-2 infection all dental treatments can be conducted under the usual safety for all parties involved.

For patients of the group B) with a negative RAT and patients of group A) who refuse to have a RAT any elective treatment should be minimized. Dental treatment should be limited to dental therapies that have been started, and need to be finished, and to emergencies only. The reasoning for this is to minimize the contact time and reduce aerosol producing treatments as far as possible. The restriction of treatment for patients in group B is also based on the fact that they should not be subjected to additional burdens due to existing signs of the disease and that RATs can be false negative, especially at a low virus load particularly shortly after infection [15].

In patients of group C) and for those patients of group B) who refuse to be tested or have a positive RAT, the treatments are limited to dental emergencies only. This follows the strategy of minimizing the contact time as well as the duration of aerosol producing treatments [16]. The patients receiving treatment would be cases under severe pain, having a bacterial infection or suffering from consequences of an accident.

For all patients it is recommended that apart from hand washing and disinfection they wear Mouth Nose protective mask, of at least (FFP) 2 quality, with mask removal only during actual intra-oral treatment.

For the dentist and the dental assistant in addition to intensive hand washing and disinfection (each $30 \mathrm{~s}$ ), use of disposable gloves and multiuse gowns, the following additional personal protection equipment (PPE) and procedures are recommended to be used for all patient groups:

- FFP2 mask.

- safety goggles / visor or face shields.

- antiseptic mouth rinse prior to treatment for 30 $60 \mathrm{~s}$ with for example $1 \% \mathrm{H}_{2} \mathrm{O}_{2}$ [3] or Listerine ${ }^{\circ}$ cool mint [17].

- use of rubber dam whenever possible.

- avoidance of using aerosol generating devices when possible,

- preference for hand instrumentation.

- intensive surface cleaning and disinfection

In addition to the above precautions, it is advised to use additional PPE (FFP2/ mask, single use gowns and bonnets etc.) with patient groups B and C [16]. Dental care providers belonging to any risk group should critically calculate the risk and balance reasons whether to treat or not to treat SARS-CoV-2 positive or suspect patients.

The focus in Table 1 is on possible measures during treatment. Furthermore, it should be considered whether protective measures for front desk staff should be taken at the entrance of the practice. It should also be considered that as few patients as possible should be in the waiting area. The time interval between two treatments in a room should be chosen sufficiently to allow disinfection measures to be carried out and to adequately ventilate. Appointments and updates should be made using digital communication [16].

\section{Discussion}

With the lifting of restrictions, normal life is returning and elective dental treatments with the above outlined risks need to be completed [19]. However, there are potentially new mutants that are classified by the WHO as variants of interest (VOI) or variants of concern (VOC), such as the lambda variant emerging in South America [20. Apart from new variants that can penetrate better or evade the immune response more easily, prevention is 
Table 1 Possibilities for clinical routine during a SARS-CoV-2 pandemic to protect patients and the dental team. It is particularly important to identify potential risks and to identify measures to minimize risks that an asymptomatic carrier infects another person directly or indirectly

\begin{tabular}{|c|c|c|c|c|c|c|c|}
\hline No & $\begin{array}{l}\text { Group/ } \\
\text { Location }\end{array}$ & Discipline & Risk & 1. Substitution & $\begin{array}{l}\text { 2. Technical } \\
\text { measures }\end{array}$ & 3. Organizational measures & $\begin{array}{l}\text { 4. Personal } \\
\text { protective } \\
\text { equipment } \\
\text { (PPE) }\end{array}$ \\
\hline \multicolumn{8}{|c|}{ Front desk } \\
\hline 1) & $\begin{array}{l}\text { Receptionist/ } \\
\text { Lobby }\end{array}$ & $\begin{array}{l}\text { Appointments, } \\
\text { conversations }\end{array}$ & $\begin{array}{l}\text { Infection risk } \\
\text { by } \\
\text { transmission } \\
\text { via droplets } \\
\text { if the } \\
\text { minimum } \\
\text { distance } \\
\text { cannot be } \\
\text { maintained. }\end{array}$ & $\begin{array}{l}\text { Clarify as much as } \\
\text { possible by phone } \\
\text { in advance }\end{array}$ & Protective screen & & $\begin{array}{l}\text { Mouth nose } \\
\text { protective } \\
\text { mask/FFP2 } \\
\text { mask }\end{array}$ \\
\hline 2) & $\begin{array}{l}\text { Receptionist/ } \\
\text { Lobby }\end{array}$ & $\begin{array}{l}\text { Receipt of patient data via } \\
\text { Chipcard }\end{array}$ & $\begin{array}{l}\text { Contact } \\
\text { transmission }\end{array}$ & Not possible & $\begin{array}{l}\text { Card reader in } \\
\text { front of screen. } \\
\text { Card handled by } \\
\text { patient. }\end{array}$ & $\begin{array}{l}\text { No handing out of chip card, } \\
\text { No physical contact, in case } \\
\text { of contact hand disinfection. }\end{array}$ & $\begin{array}{l}\text { Mouth Nose } \\
\text { protective } \\
\text { mask/FFP2 } \\
\text { mask }\end{array}$ \\
\hline \multicolumn{8}{|c|}{ Preparation work } \\
\hline 3) & $\begin{array}{l}\text { Dental } \\
\text { Assistant/ } \\
\text { Waiting } \\
\text { room/ } \\
\text { hallway }\end{array}$ & $\begin{array}{l}\text { Patients are called in and } \\
\text { guided to the Treatment } \\
\text { room }\end{array}$ & $\begin{array}{l}\text { Infection risk } \\
\text { by } \\
\text { transmission } \\
\text { via droplets } \\
\text { if the } \\
\text { minimum } \\
\text { distance is } \\
\text { not } \\
\text { maintained. }\end{array}$ & Not possible & None & $\begin{array}{l}\text { Maintain minimum distance } \\
\text { of } 1.5 \mathrm{~m} \text {. No physical contact }\end{array}$ & $\begin{array}{l}\text { Mouth Nose } \\
\text { protective } \\
\text { mask/FFP2 } \\
\text { mask }\end{array}$ \\
\hline 4) & $\begin{array}{l}\text { Dental } \\
\text { Assistant/ } \\
\text { Treatment } \\
\text { room }\end{array}$ & $\begin{array}{l}\text { Preparation work such as } \\
\text { put on a napkin, patient } \\
\text { cape }\end{array}$ & $\begin{array}{l}\text { Infection risk } \\
\text { by } \\
\text { transmission } \\
\text { via droplets } \\
\text { as the } \\
\text { minimum } \\
\text { distance } \\
\text { cannot be } \\
\text { maintained. }\end{array}$ & Not possible & None & $\begin{array}{l}1 \% \mathrm{H}_{2} \mathrm{O}_{2} \text { Mouth wash for } 60 \\
\mathrm{~s} \text { before treatment by } \\
\text { patient }\end{array}$ & $\begin{array}{l}\text { Mouth Nose } \\
\text { protective } \\
\text { mask/FFP2 } \\
\text { mask }\end{array}$ \\
\hline \multicolumn{8}{|c|}{ Diagnostic \& Non-invasive Activities } \\
\hline 5) & $\begin{array}{l}\text { Dentist/ } \\
\text { Treatment } \\
\text { room }\end{array}$ & $\begin{array}{l}\text { Activities, without invasive } \\
\text { treatment, such as } \\
\text { diagnosis consultation }\end{array}$ & $\begin{array}{l}\text { Infection risk } \\
\text { by } \\
\text { transmission } \\
\text { via droplets } \\
\text { as the } \\
\text { minimum } \\
\text { distance } \\
\text { cannot be } \\
\text { maintained } \\
{[3,4]}\end{array}$ & Not possible & None & $\begin{array}{l}1 \% \mathrm{H}_{2} \mathrm{O}_{2} \text { Mouth wash for } 60 \\
\text { s before treatment by } \\
\text { patient }\end{array}$ & $\begin{array}{l}\text { Mouth Nose } \\
\text { protective } \\
\text { mask/FFP2 } \\
\text { mask, Eye } \\
\text { protection } \\
\text { (Goggle, Visor), } \\
\text { Gloves. Caps, } \\
\text { Single use cape } \\
{[3,18]}\end{array}$ \\
\hline 6) & $\begin{array}{l}\text { Dental } \\
\text { Assistant/ } \\
\text { Dentist/ } \\
\text { Treatment } \\
\text { room }\end{array}$ & Radiograph & $\begin{array}{l}\text { Infection risk } \\
\text { by } \\
\text { transmission } \\
\text { via droplets } \\
\text { as the } \\
\text { minimum } \\
\text { distance } \\
\text { cannot be } \\
\text { maintained. }\end{array}$ & Not possible & $\begin{array}{l}\text { In patients with } \\
\text { more gagging } \\
\text { avoid optional } \\
\text { intraoral x-ray, If } \\
\text { necessary make } \\
\text { an } \\
\text { orthopantomo- } \\
\text { gram }\end{array}$ & $\begin{array}{l}1 \% \mathrm{H}_{2} \mathrm{O}_{2} \text { Mouth wash for } 60 \\
\text { s before treatment by } \\
\text { patient }\end{array}$ & $\begin{array}{l}\text { Mouth Nose } \\
\text { protective } \\
\text { mask/FFP2 } \\
\text { mask }\end{array}$ \\
\hline 7) & $\begin{array}{l}\text { Dental } \\
\text { assistant/ } \\
\text { Dentist/ } \\
\text { Treatment } \\
\text { room }\end{array}$ & $\begin{array}{l}\text { Handling of the Dental } \\
\text { impression }\end{array}$ & $\begin{array}{l}\text { Contact } \\
\text { transmission }\end{array}$ & $\begin{array}{l}\text { Not possible } \\
\text { Optional digital } \\
\text { dental impressions }\end{array}$ & $\begin{array}{l}\text { Rinse with water } \\
\text { and disinfection } \\
\text { solution }\end{array}$ & - & $\begin{array}{l}\text { Mouth Nose } \\
\text { protective } \\
\text { mask/FFP2 } \\
\text { mask, Eye } \\
\text { protection }\end{array}$ \\
\hline
\end{tabular}


Table 1 Possibilities for clinical routine during a SARS-COV-2 pandemic to protect patients and the dental team. It is particularly important to identify potential risks and to identify measures to minimize risks that an asymptomatic carrier infects another person directly or indirectly (Continued)

\begin{tabular}{|c|c|c|c|c|c|c|c|c|}
\hline No & $\begin{array}{l}\text { Group/ } \\
\text { Location }\end{array}$ & Discipline & Activities & Risk & 1. Substitution & $\begin{array}{l}\text { 2. Technical } \\
\text { measures }\end{array}$ & 3. Organizational measures & $\begin{array}{l}\text { 4. Personal } \\
\text { protective } \\
\text { equipment } \\
\text { (PPE) }\end{array}$ \\
\hline & & & & & & & & $\begin{array}{l}\text { (Goggle, Visor), } \\
\text { Gloves. Caps, } \\
\text { Single use cape } \\
{[3,18]}\end{array}$ \\
\hline \multicolumn{9}{|c|}{ Invasive Activities } \\
\hline 8) & $\begin{array}{l}\text { Dental } \\
\text { Hygienist }\end{array}$ & \multicolumn{2}{|c|}{$\begin{array}{l}\text { Prophylaxis(tooth } \\
\text { cleaning) Scaling and root } \\
\text { planning, Polishing, } \\
\text { working with rotating } \\
\text { instruments, sonic-/ } \\
\text { ultrasonic, air abrasion tool }\end{array}$} & $\begin{array}{l}\text { Infection risk } \\
\text { by } \\
\text { transmission } \\
\text { via droplets } \\
\text { and aerosols } \\
\text { as the } \\
\text { minimum } \\
\text { distance } \\
\text { cannot be } \\
\text { maintained } \\
{[3,4]}\end{array}$ & Possible & $\begin{array}{l}\text { Reduction of } \\
\text { aerosol } \\
\text { development } \\
\text { using only } \\
\text { manual } \\
\text { instruments(non } \\
\text { sonic/ ultrasonic) }\end{array}$ & Postpone to a later date, & $\begin{array}{l}\text { Mouth Nose } \\
\text { protective } \\
\text { mask/FFP2 } \\
\text { mask, Eye } \\
\text { protection } \\
\text { (Goggle, Visor), } \\
\text { Gloves. Caps, } \\
\text { Single use cape } \\
{[3,18]}\end{array}$ \\
\hline 9) & $\begin{array}{l}\text { Dental } \\
\text { Assistant/ } \\
\text { Dentist/ } \\
\text { Treatment } \\
\text { room }\end{array}$ & $\begin{array}{l}\text { Periodontal } \\
\text { initial } \\
\text { treatments/ } \\
\text { recalls }\end{array}$ & $\begin{array}{l}\text { Scaling and } \\
\text { root } \\
\text { planning, } \\
\text { working } \\
\text { with } \\
\text { rotating } \\
\text { instrument, } \\
\text { sonic-/ } \\
\text { ultrasonic, } \\
\text { air abrasion } \\
\text { tool }\end{array}$ & $\begin{array}{l}\text { Infection risk } \\
\text { by } \\
\text { transmission } \\
\text { via droplets } \\
\text { and aerosols } \\
\text { as the } \\
\text { minimum } \\
\text { distance } \\
\text { cannot be } \\
\text { maintained } \\
{[3,4]}\end{array}$ & Not possible & $\begin{array}{l}\text { Reduction of } \\
\text { Aerosol } \\
\text { development } \\
\text { using } \\
\text { predominantly } \\
\text { manual } \\
\text { instruments }\end{array}$ & $\begin{array}{l}1 \% \mathrm{H}_{2} \mathrm{O}_{2} \text { Mouth wash for } 60 \\
\mathrm{~s} \text { before treatment by } \\
\text { patient }\end{array}$ & $\begin{array}{l}\text { Mouth Nose } \\
\text { protective } \\
\text { mask/FFP2 } \\
\text { mask,Eye } \\
\text { protection } \\
\text { (Goggle, Visor), } \\
\text { Gloves. Caps, } \\
\text { Single use cape } \\
{[3,18]}\end{array}$ \\
\hline 10) & $\begin{array}{l}\text { Dental } \\
\text { Assistant/ } \\
\text { Dentist/ } \\
\text { Treatment } \\
\text { room }\end{array}$ & $\begin{array}{l}\text { Restorative } \\
\text { Treatment: } \\
\text { Direct/ } \\
\text { Indirect } \\
\text { restaurations }\end{array}$ & $\begin{array}{l}\text { working } \\
\text { with } \\
\text { rotating } \\
\text { and sonic-/ } \\
\text { ultrasonic } \\
\text { equipment }\end{array}$ & $\begin{array}{l}\text { Infection risk } \\
\text { by } \\
\text { transmission } \\
\text { via droplets } \\
\text { and aerosols, } \\
\text { as the } \\
\text { minimum } \\
\text { distance } \\
\text { cannot be } \\
\text { maintained } \\
{[3,4]}\end{array}$ & Possible & $\begin{array}{l}\text { Mouth covered } \\
\text { using the dental } \\
\text { dam. Reduction } \\
\text { of aerosol } \\
\text { development } \\
\text { minimal use of } \\
\text { sonic and } \\
\text { ultrasonic } \\
\text { instruments and } \\
\text { turbine driven } \\
\text { instruments. }\end{array}$ & $\begin{array}{l}1 \% \mathrm{H}_{2} \mathrm{O}_{2} \text { Mouth wash for } 60 \\
\mathrm{~s} \text { before treatment by } \\
\text { patient }\end{array}$ & $\begin{array}{l}\text { Mouth Nose } \\
\text { protective } \\
\text { mask/FFP2 } \\
\text { mask,Eye } \\
\text { protection } \\
\text { (Goggle, Visor), } \\
\text { Gloves. Caps, } \\
\text { Single use cape } \\
{[3,18]}\end{array}$ \\
\hline 11) & $\begin{array}{l}\text { Dental } \\
\text { Assistant/ } \\
\text { Dentist/ } \\
\text { Treatment } \\
\text { room }\end{array}$ & $\begin{array}{l}\text { Prothetic } \\
\text { Dentistry: }\end{array}$ & $\begin{array}{l}\text { working } \\
\text { with } \\
\text { rotating } \\
\text { and sonic-/ } \\
\text { ultrasonic } \\
\text { equipment }\end{array}$ & $\begin{array}{l}\text { Infection risk } \\
\text { by } \\
\text { transmission } \\
\text { via droplets } \\
\text { and aerosols, } \\
\text { as the } \\
\text { minimum } \\
\text { distance } \\
\text { cannot be } \\
\text { maintained } \\
{[3,4]}\end{array}$ & Not possible & None & $\begin{array}{l}1 \% \mathrm{H}_{2} \mathrm{O}_{2} \text { Mouth wash for } 60 \\
\mathrm{~s} \text { before treatment by } \\
\text { patient }\end{array}$ & $\begin{array}{l}\text { Mouth Nose } \\
\text { protective } \\
\text { mask/FFP2 } \\
\text { mask,Eye } \\
\text { protection } \\
\text { (Goggle, Visor), } \\
\text { Gloves. Caps, } \\
\text { Single use } \\
\text { cape[3, 18] } \\
\text { Optional prior } \\
\text { Covid test } \\
\text { recommended } \\
\text { for the patient } \\
\text { in case of larger } \\
\text { preparations } \\
\text { (several teeth) }\end{array}$ \\
\hline 12) & $\begin{array}{l}\text { Dental } \\
\text { Assistant// } \\
\text { Dentist/ } \\
\text { Treatment } \\
\text { room }\end{array}$ & $\begin{array}{l}\text { Endodontic } \\
\text { Treatment }\end{array}$ & $\begin{array}{l}\text { working } \\
\text { with } \\
\text { rotating } \\
\text { and sonic-/ } \\
\text { ultrasonic }\end{array}$ & $\begin{array}{l}\text { Infection risk } \\
\text { by } \\
\text { transmission } \\
\text { via droplets } \\
\text { and aerosols, }\end{array}$ & Not possible & $\begin{array}{l}\text { Mouth covered } \\
\text { using the dental } \\
\text { dam. } \\
\text { Desinfection of } \\
\text { the tooth prior }\end{array}$ & $\begin{array}{l}1 \% \mathrm{H}_{2} \mathrm{O}_{2} \text { Mouth wash for } 60 \\
\mathrm{~s} \text { before treatment by } \\
\text { patient }\end{array}$ & $\begin{array}{l}\text { Mouth Nose } \\
\text { protective } \\
\text { mask/FFP2 } \\
\text { mask,Eye } \\
\text { protection }\end{array}$ \\
\hline
\end{tabular}


Table 1 Possibilities for clinical routine during a SARS-CoV-2 pandemic to protect patients and the dental team. It is particularly important to identify potential risks and to identify measures to minimize risks that an asymptomatic carrier infects another person directly or indirectly (Continued)

\begin{tabular}{|c|c|c|c|c|c|c|c|c|}
\hline No & $\begin{array}{l}\text { Group/ } \\
\text { Location }\end{array}$ & Discipline & Activities & Risk & 1. Substitution & $\begin{array}{l}\text { 2. Technical } \\
\text { measures }\end{array}$ & 3. Organizational measures & $\begin{array}{l}\text { 4. Personal } \\
\text { protective } \\
\text { equipment } \\
\text { (PPE) }\end{array}$ \\
\hline & & & equipment & $\begin{array}{l}\text { as the } \\
\text { minimum } \\
\text { distance } \\
\text { cannot be } \\
\text { maintained } \\
{[3,4]}\end{array}$ & & $\begin{array}{l}\text { trepanation. } \\
\text { Reduction of } \\
\text { aerosol } \\
\text { development } \\
\text { minimal use of } \\
\text { sonic and } \\
\text { ultrasonic } \\
\text { instruments and } \\
\text { turbine driven } \\
\text { instruments. }\end{array}$ & & $\begin{array}{l}\text { (Goggle, Visor), } \\
\text { Gloves. Caps, } \\
\text { Single use } \\
\text { cape[3, } 18]\end{array}$ \\
\hline 13) & $\begin{array}{l}\text { Dental } \\
\text { Assistant/ } \\
\text { Dentist/ } \\
\text { Treatment } \\
\text { room }\end{array}$ & Orthodontics & $\begin{array}{l}\text { Brackets } \\
\text { insertion or } \\
\text { removal }\end{array}$ & $\begin{array}{l}\text { Infection risk } \\
\text { by } \\
\text { transmission } \\
\text { via droplets } \\
\text { and aerosols, } \\
\text { as the } \\
\text { minimum } \\
\text { distance } \\
\text { cannot be } \\
\text { maintained } \\
{[3,4]}\end{array}$ & Not possible & None & $\begin{array}{l}1 \% \mathrm{H}_{2} \mathrm{O}_{2} \text { Mouth wash for } 60 \\
\text { s before treatment by } \\
\text { patient }\end{array}$ & $\begin{array}{l}\text { Mouth Nose } \\
\text { protective } \\
\text { mask/FFP2 } \\
\text { mask,Eye } \\
\text { protection } \\
\text { (Goggle, Visor), } \\
\text { Gloves.Caps, } \\
\text { Single use } \\
\text { cape[3, 18] }\end{array}$ \\
\hline 14) & $\begin{array}{l}\text { Dental } \\
\text { Assistant/ } \\
\text { Dentist/ } \\
\text { Treatment } \\
\text { room }\end{array}$ & $\begin{array}{l}\text { Oral Maxillo } \\
\text { Facial } \\
\text { surgery }\end{array}$ & $\begin{array}{l}\text { working } \\
\text { with } \\
\text { cutting/ } \\
\text { oscillating/ } \\
\text { rotating } \\
\text { and sonic-/ } \\
\text { ultrasonic } \\
\text { equipment }\end{array}$ & $\begin{array}{l}\text { Infection risk } \\
\text { by } \\
\text { transmission } \\
\text { via droplets } \\
\text { and aerosols, } \\
\text { as the } \\
\text { minimum } \\
\text { distance } \\
\text { cannot be } \\
\text { maintained } \\
{[3,4]}\end{array}$ & Not possible & $\begin{array}{l}\text { Postpone } \\
\text { elective } \\
\text { therapies in } \\
\text { periods with } \\
\text { high infection } \\
\text { rates }\end{array}$ & $\begin{array}{l}1 \% \mathrm{H}_{2} \mathrm{O}_{2} \text { Mouth wash for } 60 \\
\mathrm{~s} \text { before treatment by } \\
\text { patient }\end{array}$ & $\begin{array}{l}\text { Mouth Nose } \\
\text { protective } \\
\text { mask/FFP2 } \\
\text { mask,Eye } \\
\text { protection } \\
\text { (Goggle, Visor), } \\
\text { Gloves.Caps, } \\
\text { Single use cape } \\
{[3,18] \text { Optional }} \\
\text { prior Covid test } \\
\text { recommended } \\
\text { for the patient } \\
\text { in case of } \\
\text { elective surgery } \\
\text { with increased } \\
\text { aerosol } \\
\text { developments }\end{array}$ \\
\hline
\end{tabular}

also useful, as little is known about the consequences of COVID-19 infection, currently known as long-COVID.

On the other hand, various questions need to be answered:

\section{What will be the future scenario?}

At this point it is not clear how the lifting of restrictions will affect the spreading of SARS-CoV-2. There are many open questions such as:

a) Since studies show that the anti-body level reduces over a period of time, is immunity established for vaccinated persons or persons after passing COVID-19 and how long will it prevail [21]? Are all vaccines equally effective [22]? How often do new variants require revaccination? Can memory cells be formed that trigger a humoral immune response even after a long period of illness [21]?

From the dentist perspective: Can I treat SARS-CoV-2 harboring persons without getting infected or spreading the disease myself?

From the patient perspective: Can I get SARS-CoV-2 from the dentist/dental team?

b) How to address the risk regarding the spread via non-symptomatic carriers? Studies show that especially children and young adults fall frequently in this group and help spread the virus without having any signs of the disease.

The spread of non-symptomatic carriers can be reduced by adhering to strict hygienic measures and by frequent tests. Symptomless spreaders can also be vaccinated persons. Vaccinated persons have a lower viral 
load, but the extent to which they can transmit the disease is unclear and therefore not excluded [23].

c) Will a seasonal resurgence of the virus occur causing the same problems as seen today? What effect will current variants from the UK, South Africa or Brazil and potential future variants have? Will the immunity level established be effective also against those or future variants [24]?

Because of the unanswered questions, mentioned above, a lot of uncertainties will continue to exist in the dental world. However, patients and dental providers will continue to be at risk of infection but patients need to be treated and therefore prevention of infection will continue to be a high priority in the future.

\section{How to address these risks? Identification of high-risk groups}

It is essential to protect those who are at high risk of suffering from serious sequalae from COVID-19. The definition of this risk group is constantly being expanded. Initially, people older than $60 \mathrm{y}$ or with cardiological or respiratory diseases were assumed to be at risk, but now hypertension, renal diseases, overweight people and smokers are also included. Irrespective of the ongoing definition, these patients again should critically balance the reasons whether elective dental treatment can be postponed. But the severe course of the disease may also be found in patients with no underlying preconditions and may also occur in young patients [25]. In addition, there are further groups of patients at higher risk like hemodialysis patients [26] or the group of immunosuppressed patients such as organ transplant patients or patient under immunosuppressive therapy for liver and intestinal diseases [27]. These are at risk in several respects. They have more frequent contact with doctors (practice, hospital) due to their illness, but their immune systems are no longer as efficient due to immunosuppression.

\section{Identification of carriers}

Any patient who is in general good health might also be an asymptomatic carrier of SARS-CoV-2, especially as the viral load is the highest in the early stages $[2,28]$. This poses an increased threat for all persons. Therefore, it is necessary to test as many and as frequently as possible and ideally before every treatment. In this context, it is also helpful to take a travel history and ask about infections in their immediate environment (friends, work colleagues...).

While in other contexts of daily life - such as airlines, schools, restaurants and concerts - access is only granted for those who have tested negative, the question arises, why this should not also hold true for elective dental treatment [29]? The proposed test and treatment strategy should incentivize patients, enabling a wider range of dental procedures and safeguarding emergency treatment.

While frequent testing is helpful to navigate through these times, in most countries from a legal perspective nobody - neither dentist, employees nor patients - can be forced to be tested.

The dentist is however free to offer treatment to the patient and to manage his/her staff based on the respective risk evaluation. This could also minimize potential liability risk to the dentist from patients or employees. Any test can give false positive and false negative results so the clinical assessment of COVID-19 prevalence is also important. Also, due to the reduced sensitivity of RAT compared to RT-qPCR, the test frequency is important $[30,31]$. The limitations can be overcome by increasing the test frequency [32].

To set up a RAT infrastructure within the dental practice and to perform those tests has associated costs. The dentist should be well equipped to incorporate a RAT in the practice organization. A RAT is in principle a kind of a PPE and the costs could be managed along with extra costs associated with SARS-CoV-2.

\section{Limitation of predictive value of RAT}

According to the $\mathrm{WHO}$ and European Centre for Disease Prevention and Control (ECDC), RATs have a minimum performance requirement set at $\geqq 80 \%$ sensitivity and $\geqq 97 \%$ specificity, which is lower than those for RT-qPCR tests [33]. They perform best in cases with high viral load, in presymptomatic and early symptomatic cases. This means that in the very early stages of the infection they don't perform as well. The results of the RAT have always to be seen in conjunction with the actual prevalence settings. In high prevalence settings RAT will have a high positive predictive value (PPV) and low negative predictive values NPV [34]. If you take for example a test with a sensitivity of $80 \%$ and a specificity of $98 \%$ and a prevalence of $20 \%$ the PPV is 0.909 and the NPV is 0.951 or in other words 9 out of 100 have a false positive result and 5 out of 100 have a false negative result. While at $10 \%$ prevalence the values are PPV $=0.816$ and $\mathrm{NPV}=0.978$ respectively. Here 18 out of 100 might have a false positive result and 2 out of 100 a false negative result. At $1 \%$ prevalence the rates for PPV are 0.288 and for NPV are 0.998 . In 71 out of 100 cases the positive result is false and 2 out of 1000 cases show a false negative result. This means that in settings of high prevalence a positive test result will be very likely be true. In low prevalence settings a negative test is likely to be true, whereas a positive RAT should, due to the higher accuracy of RT-qPCR tests, always be confirmed with this test system. In general RATs can help to reduce transmission through early detection of highly infectious cases and enable a rapid start of contact tracing.

It is also unclear whether vaccinated persons or persons who have been infected are "safe" [35]. Recent studies show that the antibody level diminishes over time, so 
that it remains unclear how long this protection might actually last. Cases are described in the literature where even vaccinated persons can become re-infected [36].

Tracing apps can help identify people being exposed to an infected person [37]. But their effectiveness remains to be proven. Therefore, practical and workable measures must be developed and implemented so RATs may be a helpful adjunct to establish a safer daily routine. The advantage of RATs, apart from low cost, is their availability and flexibility, requiring no infrastructure, their ease of handling and rapid results.

The limitation of this study is that the test and treatment strategy for the operation of a dental practice during SARS-CoV-2 prevalence is based on the effectiveness of RAT to detect the prevailing variants. Another limitation is that there are no decision-making guidelines, as for other infectious diseases, that can be adapted for (dental) practices within the framework of the work.

To incorporate RAT in the dental practice, aspects like additional time requirements, the incorporation of the test results in the practice workflow, additional and trained staff, space requirements need to be addressed as well as the question about the associated additional costs.

\section{Conclusions}

The fairly low incidence of infected dentists may indicate that dentists who are used to stringent hygienic standards and use of them consistently are not at higher risk. However, further studies need to confirm that observation. Given the wide scope of dentistry, those studies should not only include the number of dentists tested positive compared to other medical groups, but also consider the specific variables of the various disciplines (dental prosthetics, orthodontics, oralmaxillofacial surgery, periodontology, endodontics, restorative dentistry etc.) with different aerosol producing activities or possibility using rubber dam. Until those results are available, it appears to be imperative to maintain or further improve on those standards [3].

As the virus will be present in the foreseeable future it is necessary to establish safe routines for the dentist, his/ her staff, and the patients to identify the respective carriers and to offer treatments based on the specific risk assessment. RATs, despite their limitations, could help the dentist manage the situation.

Finally, these measures are an additional cost to the practice if not reimbursed. Nevertheless, no savings should be made on security.

\footnotetext{
Abbreviations

COVID-19: Corona Virus Disease 2019; ECDC: European Centre for Disease Prevention and Control; FFP: Filtering Face Piece; NPV: Negative Predictive Value; POC: Point of Care; PPE: Personal Protection Equipment; PPV: Positive Predictive Value; RAT: Rapid Antigen Test; RT-qPCR: Reverse Transcriptionquantitative polymerase chain reaction; s: Second; SARS-CoV-2: Severe acute respiratory syndrome coronavirus 2; WHO: World Health Organization; y: Year
}

\section{Authors' contributions}

All authors have developed the concept and studied the relevant literature. M. Draenert and T. Beikler focused on patient aspects; J. Durner, D.C. Watts and M. Becker on the analytical aspects. The author(s) read and approved the final manuscript

\section{Funding}

The work was not supported by any society/ company/ industry. Open Access funding enabled and organized by Projekt DEAL.

\section{Availability of data and materials}

All data and their sources are clearly shown.

\section{Declarations}

\section{Ethics approval and consent to participate}

This article does not contain any studies with human participants or animals performed by any of the authors.

Consent for publication

All authors declare their consent.

\section{Competing interests}

All authors declare that they have no conflict of interest.

\section{Author details}

${ }^{1}$ Department of Conservative Dentistry and Periodontology, University Hospital, LMU Munich, Ludwig-Maximilians-University Munich, Goethestr. 70, Goethestraße 70, 80336 Munich, Germany. ${ }^{2}$ Laboratory Becker \& Colleagues, Führichstr. 70, 81671 Munich, Germany. ${ }^{3}$ Department of Periodontics, Preventive and Restorative Dentistry, University Medical Center Hamburg-Eppendorf, Martinistraße 52 (Building O58), 20246 Hamburg, Germany. ${ }^{4}$ School of Medical Sciences and Photon Science Institute, University of Manchester, Manchester, UK.

Received: 1 June 2021 Accepted: 24 August 2021

Published online: 04 September 2021

\section{References}

1. Sabino-Silva R, Jardim ACG, Siqueira WL. Coronavirus COVID-19 impacts to dentistry and potential salivary diagnosis. Clin Oral Investig. 2020;24:161921. https://doi.org/10.1007/s00784-020-03248-x.

2. Meng L, Hua F, Bian Z. Coronavirus Disease 2019 (COVID-19): Emerging and Future Challenges for Dental and Oral Medicine. J Dent Res. 2020: 22034520914246. https://doi.org/10.1177/0022034520914246.

3. $\quad$ Peng X, Xu X, Li Y, Cheng L, Zhou X, Ren B. Transmission routes of 2019nCoV and controls in dental practice. Int J Oral Sci. 2020;12:9. https://doi. org/10.1038/s41368-020-0075-9.

4. Bentley CD, Burkhart NW, Crawford JJ. Evaluating spatter and aerosol contamination during dental procedures. J Am Dent Assoc. 1994;125:57984. https://doi.org/10.14219/jada.archive.1994.0093.

5. Grenier D. Quantitative analysis of bacterial aerosols in two different dental clinic environments. Appl Environ Microbiol. 1995;61:3165-8.

6. Hartley GE, Edwards ESJ, Aui PM, Varese N, Stojanovic S, McMahon J, et al. Rapid generation of durable B cell memory to SARS-CoV-2 spike and nucleocapsid proteins in COVID-19 and convalescence. Sci Immunol. 2020;5. https://doi.org/10.1126/sciimmunol.abf8891.

7. Yamayoshi S, Sakai-Tagawa Y, Koga M, Akasaka O, Nakachi I, Koh H, et al. Comparison of Rapid Antigen Tests for COVID-19. Viruses. 2020;12. https:// doi.org/10.3390/v12121420.

8. Durner J, Burggraf S, Czibere L, Fleige T, Madejska A, Watts DC, et al. Fast and simple high-throughput testing of COVID 19. Dent Mater. 2020. https:// doi.org/10.1016/j.dental.2020.04.001.

9. Durner J, Burggraf S, Czibere L, Tehrani A, Watts DC, Becker M. Fast and cost-effective screening for SARS-CoV-2 variants in a routine diagnostic setting. Dent Mater. 2021. https://doi.org/10.1016/j.dental.2021.01.015.

10. Bruning $A H L$, Leeflang MMG, Vos J, Spijker R, de Jong MD, Wolthers KC, et al. Rapid Tests for Influenza, Respiratory Syncytial Virus, and Other Respiratory Viruses: A Systematic Review and Meta-analysis. Clin Infect Dis. 2017;65:1026-32. https://doi.org/10.1093/cid/cix461. 
11. Scohy A, Anantharajah A, Bodéus M, Kabamba-Mukadi B, Verroken A, Rodriguez-Villalobos H. Low performance of rapid antigen detection test as frontline testing for COVID-19 diagnosis. J Clin Virol. 2020;129:104455. https://doi.org/10.1016/j.jcv.2020.104455

12. Bohn MK, Lippi G, Horvath AR, Erasmus R, Grimmler M, Gramegna M, et al. IFCC interim guidelines on rapid point-of-care antigen testing for SARS-CoV2 detection in asymptomatic and symptomatic individuals. Clin Chem Lab Med. 2021. https://doi.org/10.1515/cclm-2021-0455.

13. Arons MM, Hatfield KM, Reddy SC, Kimball A, James A, Jacobs JR, et al. Presymptomatic SARS-CoV-2 Infections and Transmission in a Skilled Nursing Facility. N Engl J Med. 2020;382:2081-90. https://doi.org/10.1056/ NEJMoa2008457.

14. Boyarsky BJ, Werbel WA, Avery RK, Tobian AAR, Massie AB, Segev DL, et al. Antibody Response to 2-Dose SARS-CoV-2 mRNA Vaccine Series in Solid Organ Transplant Recipients. JAMA. 2021;325:2204-6. https://doi.org/10.1 001/jama.2021.7489.

15. Blairon L, Cupaiolo R, Thomas I, Piteus S, Wilmet A, Beukinga I, et al. Efficacy comparison of three rapid antigen tests for SARS-CoV-2 and how viral load impact their performance. J Med Virol. 2021. https://doi. org/10.1002/jmv.27108.

16. Diegritz C, Manhart J, Bucher K, Grabein B, Schuierer G, Kuhnisch J, et al. A detailed report on the measures taken in the Department of Conservative Dentistry and Periodontology in Munich at the beginning of the COVID-19 outbreak. Clin Oral Investig. 2020;24:2931-41. https://doi.org/10.1007/ s00784-020-03440-z.

17. Meister TL, Bruggemann Y, Todt D, Conzelmann C, Muller JA, Gross R, et al. Virucidal Efficacy of Different Oral Rinses Against Severe Acute Respiratory Syndrome Coronavirus 2. J Infect Dis. 2020;222:1289-92. https://doi.org/10.1 093/infdis/jiaa471

18. Baghizadeh Fini M. What dentists need to know about COVID-19. Oral Oncol. 2020;105:104741. https://doi.org/10.1016/j.oraloncology.2020.104741.

19. Marui VC, Souto MLS, Rovai ES, Romito GA, Chambrone L, Pannuti CM. Efficacy of preprocedural mouthrinses in the reduction of microorganisms in aerosol: A systematic review. J Am Dent Assoc. 2019;150:1015-26 e1. https://doi.org/10.1016/j.adaj.2019.06.024.

20. World Health O. COVID-19 weekly epidemiological update, edition 44, 15 June 2021. Geneva: World Health Organization; 2021.

21. Turner JS, Kim W, Kalaidina E, Goss CW, Rauseo AM, Schmitz AJ, et al. SARSCoV-2 infection induces long-lived bone marrow plasma cells in humans. Nature. 2021. https://doi.org/10.1038/s41586-021-03647-4.

22. He Q, Mao Q, An C, Zhang J, Gao F, Bian L, et al. Heterologous prime-boost: breaking the protective immune response bottleneck of COVID-19 vaccine candidates. Emerg Microbes Infect. 2021;10:629-37. https://doi.org/10.1 080/22221751.2021.1902245.

23. Levine-Tiefenbrun M, Yelin I, Katz R, Herzel E, Golan Z, Schreiber L, et al. Initial report of decreased SARS-CoV-2 viral load after inoculation with the BNT162b2 vaccine. Nat Med. 2021;27:790-2. https://doi.org/10.1038/s41591021-01316-7.

24. Lustig Y, Zuckerman N, Nemet I, Atari N, Kliker L, Regev-Yochay G, et al. Neutralising capacity against Delta (B.1.617.2) and other variants of concern following Comirnaty (BNT162b2, BioNTech/Pfizer) vaccination in health care workers, Israel. Euro Surveill. 2021;26. https://doi.org/10.2807/1560-7917.ES.2 021.26.26.2100557

25. Huang C, Wang Y, Li X, Ren L, Zhao J, Hu Y, et al. Clinical features of patients infected with 2019 novel coronavirus in Wuhan, China. Lancet. 2020;395:497-506. https://doi.org/10.1016/S0140-6736(20)30183-5.

26. Broseta JJ, Rodriguez-Espinosa D, Rodriguez N, Mosquera MDM, Marcos MA Egri N, et al. Humoral and Cellular Responses to mRNA-1273 and BNT162b2 SARS-CoV-2 Vaccines Administered to Hemodialysis Patients. Am J Kidney Dis. 2021. https://doi.org/10.1053/j.ajkd.2021.06.002.

27. Neurath MF. COVID-19: biologic and immunosuppressive therapy in gastroenterology and hepatology. Nat Rev Gastroenterol Hepatol. 2021. https://doi.org/10.1038/s41575-021-00480-y.

28. Rothe C, Schunk M, Sothmann P, Bretzel G, Froeschl G, Wallrauch C, et al. Transmission of 2019-nCoV Infection from an Asymptomatic Contact in Germany. N Engl J Med. 2020;382:970-1. https://doi.org/10.1056/NEJMc2 001468

29. Kiang MV, Chin ET, Huynh BQ, Chapman LAC, Rodriguez-Barraquer Greenhouse B, et al. Routine asymptomatic testing strategies for airline travel during the COVID-19 pandemic: a simulation study. Lancet Infect Dis. 2021. https://doi.org/10.1016/S1473-3099(21)00134-1.
30. Seynaeve Y, Heylen J, Fontaine C, Maclot F, Meex C, Diep AN, et al. Evaluation of Two Rapid Antigenic Tests for the Detection of SARS-CoV-2 in Nasopharyngeal Swabs. Journal of clinical medicine. 2021;10. https://doi. org/10.3390/jcm10132774.

31. Korenkov M, Poopalasingam N, Madler M, Vanshylla K, Eggeling R, Wirtz M, et al. Evaluation of a rapid antigen test to detect SARS-CoV-2 infection and identify potentially infectious individuals. J Clin Microbiol. 2021:JCM0089621. https://doi.org/10.1128/JCM.00896-21.

32. Larremore DB, Wilder B, Lester E, Shehata S, Burke JM, Hay JA, et al. Test sensitivity is secondary to frequency and turnaround time for COVID-19 screening. Sci Adv. 2021;7. https://doi.org/10.1126/sciadv.abd5393.

33. Van Walle I, Leitmeyer K, Broberg EK. Meta-analysis of the clinical performance of commercial SARS-CoV-2 nucleic acid, antigen and antibody tests up to 22 August 2020. medRxiv. 2020:2020.09.16.20195917. https://doi. org/10.1101/2020.09.16.20195917.

34. Watson J, Whiting PF, Brush JE. Interpreting a covid-19 test result. BMJ. 2020;369:m1808. https://doi.org/10.1136/bmj.m1808.

35. Anand SP, Prevost J, Nayrac M, Beaudoin-Bussieres G, Benlarbi M, Gasser R, et al. Longitudinal analysis of humoral immunity against SARS-CoV-2 Spike in convalescent individuals up to 8 months post-symptom onset. bioRxiv. 2021. https://doi.org/10.1101/2021.01.25.428097.

36. Keehner J, Horton LE, Pfeffer MA, Longhurst CA, Schooley RT, Currier JS, et al. SARS-CoV-2 Infection after Vaccination in Health Care Workers in California. N Engl J Med. 2021:384:1774-5. https://doi.org/10.1056/NEJMc2101927.

37. Ferretti L, Wymant C, Kendall M, Zhao L, Nurtay A, Abeler-Dorner L, et al. Quantifying SARS-CoV-2 transmission suggests epidemic control with digital contact tracing. Science. 2020;368. https://doi.org/10.1126/science.abb6936.

\section{Publisher's Note}

Springer Nature remains neutral with regard to jurisdictional claims in published maps and institutional affiliations.
Ready to submit your research? Choose BMC and benefit from:

- fast, convenient online submission

- thorough peer review by experienced researchers in your field

- rapid publication on acceptance

- support for research data, including large and complex data types

- gold Open Access which fosters wider collaboration and increased citations

- maximum visibility for your research: over $100 \mathrm{M}$ website views per year

At BMC, research is always in progress.

Learn more biomedcentral.com/submissions 